\title{
Outcome of a single XEN microstent implant for glaucoma patients with different types of glaucoma
}

Marc Schargus ${ }^{1}$, Theresa Theilig², Matus Rehak ${ }^{2}$, Catharina Busch², Caroline Bormann ${ }^{2}$ and Jan Darius Unterlauft ${ }^{2 *}$

\begin{abstract}
Background: The aim of this retrospective study was to compare the efficacy and safety profile of a single XENmicrostent in different types of primary and secondary open angle glaucoma.

Methods: A single XEN microstent was implanted in patients with primary open-angle glaucoma (POAG), normaltension glaucoma (NTG), pseudoexfoliation glaucoma (PEX) and secondary glaucoma (Sec.Gl). The intraocular pressure (IOP), the active substances of the applied IOP-lowering drugs, the best corrected visual acuity (BCVA) and the mean deviation (MD) of the perimetry were measured at baseline and at regular follow-ups, scheduled at 2 days and 1, 3, 6 and 12 months after surgery.

Results: 153 eyes were included in this analysis. 113 eyes were affected by POAG (74\%), 5 eyes by NTG (3\%), 22 eyes by PEX (14\%) and 13 eyes by Sec. Gl (9\%). Mean IOP decreased in all treatment groups during the 12 months of follow-up (complete group: $23.9 \pm 7.4$ to $15.4 \pm 5.1 \mathrm{mmHg}(p<0.01)$ ) POAG: $22.8 \pm 6.5$ to $15.1 \pm 4.6 \mathrm{mmHg}(p<$ 0.01); NTG: $16.6 \pm 3.4$ to $11.6 \pm 2.2 \mathrm{mmHg}(p<0.05)$; PEX: $28.0 \pm 7.9$ to $17.1 \pm 6.6 \mathrm{mmHg}(p<0.01)$; Sec.Gl: $28.9 \pm 13.9$ to $15.5 \pm 6.9 \mathrm{mmHg}(p<0.05))$. In the 153 eyes the average number of IOP-lowering drugs applied decreased from $2.6 \pm 1.2$ to $0.8 \pm 1.312$ months after surgery $(p<0.01)$. BCVA and mean deviation of automated standard perimetry remained stable in all groups during follow-up.

Conclusion: As in eyes suffering from POAG, IOP and number of IOP-lowering drugs applied can be effectively reduced by XEN implantation in eyes suffering from NTG, PEX and secondary glaucoma while leaving BCVA and visual field unchanged.
\end{abstract}

Trial registration: Trial was registered at DRKS (registration number: DRKS00020800, Registered 25.February 2020 Retrospectively registered).

Keywords: Primary open-angle Glaucoma, Trabeculectomy, XEN, MIGS

\footnotetext{
* Correspondence: jandarius.unterlauft@medizin.uni-leipzig.de

${ }^{2}$ Department of Ophthalmology, University Eye Hospital, University of Leipzig, Liebigstrasse 10-14, 04103 Leipzig, Germany

Full list of author information is available at the end of the article
}

(c) The Author(s). 2020 Open Access This article is licensed under a Creative Commons Attribution 4.0 International License, which permits use, sharing, adaptation, distribution and reproduction in any medium or format, as long as you give appropriate credit to the original author(s) and the source, provide a link to the Creative Commons licence, and indicate if changes were made. The images or other third party material in this article are included in the article's Creative Commons. licence, unless indicated otherwise in a credit line to the material. If material is not included in the article's Creative Commons licence and your intended use is not permitted by statutory regulation or exceeds the permitted use, you will need to obtain permission directly from the copyright holder. To view a copy of this licence, visit http://creativecommons.org/licenses/by/4.0/ The Creative Commons Public Domain Dedication waiver (http://creativecommons.org/publicdomain/zero/1.0/) applies to the data made available in this article, unless otherwise stated in a credit line to the data. 


\section{Background}

Glaucoma is one of the leading causes of blindness worldwide, with an estimated prevalence of $2-4 \%$ in the 40 years and older age group [1,2]. Glaucoma is a very heterogeneous group of diseases characterized by progressive atrophy of the optic disc caused by apoptotic retinal ganglion cell death $[3,4]$. Known risk factors for the development of glaucoma are an elevated intraocular pressure (IOP), familial history of glaucoma, myopia and thin central corneal thickness [5-7]. Reduction of IOP by means of medication and/or surgery is the only known way to effectively slow down further disease progression $[8,9]$.

Usually, IOP reduction by means of medication is the first-line treatment in glaucoma therapy. Surgical intervention is indicated in cases of insufficient medical IOP reduction, problems with side effects, insufficient adherence and persistence to therapy and further progression of optic nerve damage [10].. Trabeculectomy (TE) with or without the use of antimetabolites was first introduced in the mid-1960s and has since remained the "gold standard" for long-term surgical IOP reduction $[11,12]$. However, TE has numerous risks and unintended side effects that limit its use to advanced disease stages [13-16]. In recent years, various techniques have been developed to minimize surgical trauma and the risk of serious side effects. These newly developed techniques make use of different mechanisms to reduce the resistance of aqueous humor outflow, thereby reducing IOP. These new techniques are summarized under the term minimally invasive glaucoma surgery (MIGS) [17].

A promising MIGS procedure is the XEN microstent. The XEN microstent is approved for the treatment of primary open-angle glaucoma (POAG). The XEN microstent facilitates subconjunctival drainage of aqueous humor from the anterior chamber, which is comparable to the mode of action utilized in TE [18]. The XEN microstent does not comprise a valve mechanism, but maintains a minimum IOP of approximately $8 \mathrm{mmHg}$ through its length and internal lumen [19].

XEN microstent studies reported in the literature are very heterogeneous in terms of design, glaucoma entity, inclusion and exclusion criteria, preoperative pressure and type of analysis. XEN microstent significantly reduced IOP and medication use as a solo or as a combined procedure in most glaucoma entities but number of patients are low in other than POAG. Significant differences in study results may be due to the way data have been analyzed or different statistical problems due to follow up loss [20].

However, subconjunctival drainage of aqueous humor is suitable for most types of glaucoma to lower or stop visual field progression rate. Therefore, the concept of this study was to analyze the IOP-lowering efficacy of the XEN microstent in the treatment also of other types of glaucoma. Other glaucoma entities suitable for XEN implantation were pseudoexfoliation glaucoma (PEX), secondary open-angle glaucoma of various causes (Sec.Gl) and normal tension glaucoma (NTG). The main objective of the investigations was to analyze whether or not the XEN implant is also suitable for the treatment of these types of glaucoma. Furthermore, all important parameters such as visual field progression, needling rate, changes in medication, complications and visual acuity development were to be investigated in all patients over the entire 12-month period, with 12-month data being available for all patients.

\section{Methods}

The medical records of all patients/eyes scheduled for XEN microstent implantation from January 1, 2017 to December 31, 2018 at the University Eye Hospital in Leipzig were analyzed for this retrospective study.

The study was approved by the local ethics committee of the University Leipzig, Leipzig, Germany. Written informed consent was obtained from all patients. All procedures performed met the ethical standards of the institutional research commission as well as the Helsinki Declaration of 1964 and its later amendments. The trial was registered with the DRKS (registration number: DRKS00020800, Registered 25. February 2020 - Retrospectively registered,https://www.drks.de/drks_web/ navigate.do? navigationId=trial. HTML\&TRIAL_ID = DRKS00020800).

Signs of optic disc changes such as an increased cup/ disc ratio, optic disc hemorrhages, nerve fiber layer defects and/or indentations of the papillary vessels verified glaucoma diagnosis. Untreated IOP had to be $\geq 21$ $\mathrm{mmHg}$ in POAG, PEX and secondary glaucoma cases. In the cases analyzed, surgical intervention was necessary due to disease progression or medically uncontrollable IOP. In NTG IOP was by definition lower than 21 $\mathrm{mmHg}$ and disease progression alone was the single indication for surgery. This was demonstrated by deterioration of repeated automated standard perimetry results and/or reduction of retinal nerve fiber layer (RNFL) thickness on repeated optical coherence tomography (RNFL-OCT) examinations. Topical IOP-lowering therapy was discontinued 4 weeks prior to surgery in eyes scheduled for XEN implantation and preservative-free steroid eye drops were administered four times daily to achieve an irritation free conjunctiva. Additionally systemically acting acetazolamide ( $250 \mathrm{mg}$ b.i.d.) was started 4 weeks before surgery to prevent IOP spikes. Exclusion criteria were a patient age below 40 years and a narrow anterior chamber angle. If both eyes of a single patient required surgical treatment for glaucoma, only the 
results of the first-operated eye were included into this analysis.

Four weeks prior to surgery, a ophthalmological examination was performed with anti-glaucomatous local medication. This included measurement of bestcorrected visual acuity (BCVA) (Snellen charts converted to $\log M A R$ ), objective refraction, slit-lamp examination with evaluation of the optic disc by indirect ophthalmoscopy and RNFL-OCT scans; Spectralis, Heidelberg Engineering, Heidelberg, Germany), automated standard perimetry (Twinfield 2, Oculus, Wetzlar, Germany; 24-2 test strategy, 55 targets), IOP measurement with Goldmann applanation tonometry and gonioscopy with the Sussmann four-mirror contact lens. The indication for the planned surgical procedure was re-evaluated, whereby the aim was to lower IOP and/or to reduce the number of necessary IOP-lowering agents. The demographic patient data collected prior to surgery were age, sex and laterality of the operated eye. The surgical procedure included only single XEN microstent implantation. None of the included cases were combined with phacoemulsification and IOL implantation.

\section{Surgical technique}

The surgical technique used has already been described in previous publications of the group [21]. In short, two paracenteses were applied and the anterior chamber was filled with a dispersive viscoelastic agent. $0.1 \mathrm{ml} 0.01 \%$ mitomycin C (MMC) was administered subconjunctivally. The XEN microstent injector was then passed through the sclera ab interno and excised subconjunctivally. The XEN stent was then implanted. Where necessary, the stent tip was straightened with a $30 \mathrm{G}$ needle as part of a primary needling procedure.

Postoperative visits were scheduled at day 1 and 2 as well as at month 1, 3 and 6 and 12 after surgery. All patients were examined at all examination dates. All local and oral antiglaucoma medications were discontinued. Preservative-free steroid eye drops were given 6 times daily for 4 weeks and then slowly reduced over 3 months. In cases with suspected steroid-induced IOP increase, which was usually encountered at the follow-up visit 1 month after surgery, steroids were reduced faster than usual over the next 3 weeks and additional IOP-lowering agents were applied. IOP lowering compounds used were usually adapted to the therapy given before surgery when tolerated. If IOP increase was due to conjunctival scaring with absence of a visible bleb / conjunctival filtration zone at any time point during follow-up a needling procedure was scheduled. Additionally preservativefree antibiotic eye drops were prescribed four times daily for 1 week. At each visit BCVA and IOP were measured and the anterior and posterior eye segments were evaluated for adverse events (conjunctival scarring, flat bleb, shallow anterior chamber, choroidal detachment, hypotony maculopathy etc.). The number of glaucoma medications taken was queried. In addition, visual field examinations were performed during the visits 6 and 12 months after the surgery.

For additional necessary post-surgical needling procedures, a 27G cannula was used on a syringe with 5Fluoruracil (5-FU) $(5 \mathrm{mg}$ in $0.1 \mathrm{ml})$. The needle was inserted under the conjunctiva $3 \mathrm{~mm}$ temporal from the XEN stent and needling was performed by sweeping the needle tip posteriorly above and beneath the XEN microstent. Finally $0.1 \mathrm{ml}$ of 5 -FU was injected posterior to the end of the XEN microstent.

Clinical success was defined according to the recommendations of the World Glaucoma Association [22]. For complete success (A) an IOP reduction of $\geq 20 \%$ from baseline and resulting IOP of $<21 \mathrm{mmHg}$ without the use of IOP-lowering drugs had to be achieved. For qualified success (A) the same criteria applied as for complete success A with the use of additional IOPlowering drugs permitted. For complete success (B) an IOP reduction of $\geq 30 \%$ from baseline and resulting IOP of $<18 \mathrm{mmHg}$ without the use of IOP-lowering drugs had to be achieved. For qualified success (B) the same criteria applied as for complete success B with the use of additional IOP-lowering drugs permitted. For complete success $(C)$ an IOP reduction of $\geq 40 \%$ from baseline and resulting IOP of $<15 \mathrm{mmHg}$ without the use of IOPlowering drugs had to be achieved. For qualified success (C) the same criteria applied as for complete success C with the use of additional IOP-lowering drugs permitted.

Data collection and statistical analysis were performed using Excel (Version 2007, Microsoft; Redmond, USA) and SPSS (IBM Version 22.0; Chicago, Illinois, USA). The tested indices for patient age, BCVA, objective refraction and visual field are given as mean and standard deviation. The differences between the pre- and postoperative results for IOP, IOP reduction, BCVA, number of active IOP-lowering agents applied and the mean defect of static automated perimetry were analyzed using the Kruskal-Wallis nonparametric test or the MannWhitney test (where applicable) for intergroup comparisons and the Friedman test for intragroup comparisons of results measured in groups of different glaucoma types. A $p \leq 0.05$ indicated statistical significance.

\section{Results}

For this analysis the clinical course of 153 eyes of 153 patients (80 female and 73 male; 77 right and 76 left eyes) were included. Four groups of eyes suffering from different types of glaucoma were treated. 113 eyes (74\%) were treated for POAG, 5 eyes (3\%) for NTG, 13 eyes (9\%) for secondary glaucoma and 22 eyes (14\%) for pseudoexfoliation glaucoma. Of the 13 secondary 
glaucoma cases, four were due to previous eye trauma, four were due to neovascularization ( 2 cases of proliferative diabetic eye disease and 2 cases of central retinal vein occlusion, the underlying disease being considered in a stable phase at the time of XEN implantation), three cases were secondary to uveitis and the last two cases were due to steroid-induced glaucoma. In the complete patient group the mean age of patients at the time of surgery was $70.2 \pm 10.8$ years. The difference in mean patient age was not statistically significant between the four treated patient groups $(p=0.24)$. Before surgery the mean IOP of the 153 eyes was $23.9 \pm 7.4 \mathrm{mmHg}$ in the 153 eyes. Statistical analysis revealed significant IOP differences between the four subgroups (Kruskal-Wallis: $p<0.01$ ). Further analysis revealed differences of statistical significance when comparing IOP results between POAG and PEX groups $(p=0.001)$, NTG and PEX groups $(p=0.001)$ and NTG and secondary glaucoma groups $(p=0.02)$. The mean number of active substances contained in the applied IOP-lowering eye drops was $2.6 \pm 1.2$ before surgery, and the comparison between the four treated groups showed no statistically significant difference (Kruskal-Wallis: $p=0.56$ ). The mean defect tested by standard automated perimetry was $10.1 \pm$ $4.3 \mathrm{~dB}$ before surgery, and the comparison between the four treated groups showed no statistically significant difference (Kruskal-Wallis: $p=0.96$ ). The exact demographic data are also presented in Table 1.

Table 1 Patient demographic data

\begin{tabular}{ll}
\hline age [years] & 70.2 $\pm 10.8(p=0.24)$ \\
& POAG: $70.5 \pm 8.5$ \\
& NTG: $76.6 \pm 3.9$ \\
& PEX: $73.9 \pm 5.9$ \\
& Secondary Glaucoma: $68.7 \pm 10.7$ \\
& 77 right; 76 left \\
laterality & 80 female; 73 male \\
gender & POAG: 113 eyes (74\%) \\
diagnosis & NTG: 5 eyes (3\%) \\
& PEX: 22 eyes (14\%) \\
& Secondary glaucoma: 13 eyes (9\%) \\
& $\mathbf{2 . 6} \pm \mathbf{1 . 2}(p=0.56)$ \\
number of medications [n] & POAG: $2.5 \pm 1.2$ \\
& NTG: $2.6 \pm 0.9$ \\
& PEX: $2.9 \pm 1.2$ \\
& Secondary Glaucoma: $2.7 \pm 0.9$ \\
& $10.1 \pm 4.3(p=0.96)$ \\
& POAG: $10.2 \pm 3.9$ \\
& NTG: $10.8 \pm 2.9$ \\
mean deviation [dB] & PEX: $9.7 \pm 4.7$ \\
& Secondary Glaucoma: $10.1 \pm 6.8$
\end{tabular}

Patient demographic data for the 153 eyes treated with XEN microstent implantation in uncontrolled glaucoma. Also shown are the glaucoma types and the percentage of each glaucoma type as a proportion of the total treated eye group

\section{IOP results}

In the complete group of eyes analyzed mean IOP before surgery was $23.9 \pm 7.4 \mathrm{mmHg}$. Two days after XEN implantation, mean IOP decreased to $9.2 \pm 5.2 \mathrm{mmHg}$ and then increased again to $15.5 \pm 7.9 \mathrm{mmHg} 1$ month and to $15.9 \pm 6.1 \mathrm{mmHg} 3$ months after surgery. Thereafter, mean IOP remained stable with a mean value of $15.4 \pm$ $5.1 \mathrm{mmHg} 1$ year after XEN implantation, which corresponds to a mean IOP reduction of $31 \%$ compared to baseline before surgery. For the complete group of eyes analyzed the difference between the mean IOP results measured at all follow-up examinations and those measured at baseline was statistically significant at all-time points $(p<0.01)$. The exact values and the course of mean IOP during the first 12 months after surgery for the complete group as well as for the four treated subgroups are summarized in Figs. $1 \& 2$ and Table 2.

\section{IOP results in different types of glaucoma}

Mean IOP at baseline before XEN implantation in the 113 POAG eyes was $22.8 \pm 6.5 \mathrm{mmHg}$. Two days after surgery mean IOP dropped to $8.9 \pm 5.1 \mathrm{mmHg}$. From the follow-up examination 1 month after surgery the IOP remained stable at about $15 \mathrm{mmHg}$ and 1 year postoperatively the mean value was $15.1 \pm 4.6 \mathrm{mmHg}$. This corresponds to an average IOP decrease of $31 \%$ compared to baseline. Comparison of mean IOP results before and after surgery showed a difference of statistical significance $(p<0.01)$ at all follow-up examinations.

In the 5 NTG eyes the mean IOP before XEN implantation was $16.6 \pm 3.4 \mathrm{mmHg}$. Mean IOP decreased to $5.6 \pm 2.7 \mathrm{mmHg} 2$ days after surgery and rose again to $10.0 \pm 3.0 \mathrm{mmHg} 1$ month after surgery and remained relatively stable thereafter with a mean value of $11.6 \pm$ $2.2 \mathrm{mmHg} 12$ months after surgery. This corresponds to an IOP reduction of $29 \%$ from baseline. IOP was well regulated, with all five eyes achieving an $\mathrm{IOP}<15 \mathrm{mmHg}$ 12 months after surgery. Comparison of mean IOP results before and after surgery showed a difference of statistical significance $(p<0.05)$ at all follow-up examinations.

In the 22 treated eyes suffering from PEX mean IOP before surgery was $28.0 \pm 7.9 \mathrm{mmHg}$. Similarly to the other treatment groups mean IOP decreased to $11.9 \pm$ $6.4 \mathrm{mmHg} 2$ days after surgery and increased to $16.5 \pm$ $8.0 \mathrm{mmHg} 1$ month after surgery. Thereafter, IOP remained reasonably stable with a mean value of $17.1 \pm$ $6.6 \mathrm{mmHg} 1$ year after XEN implantation, which corresponds to a decrease of $38 \%$ compared to baseline. The comparison between the average IOP results before and after surgery showed a difference of statistical significance $(p<0.01)$ at all follow-up examinations.

In the 13 eyes suffering from secondary glaucoma mean IOP before surgery was $28.9 \pm 13.9 \mathrm{mmHg}$. Mean 

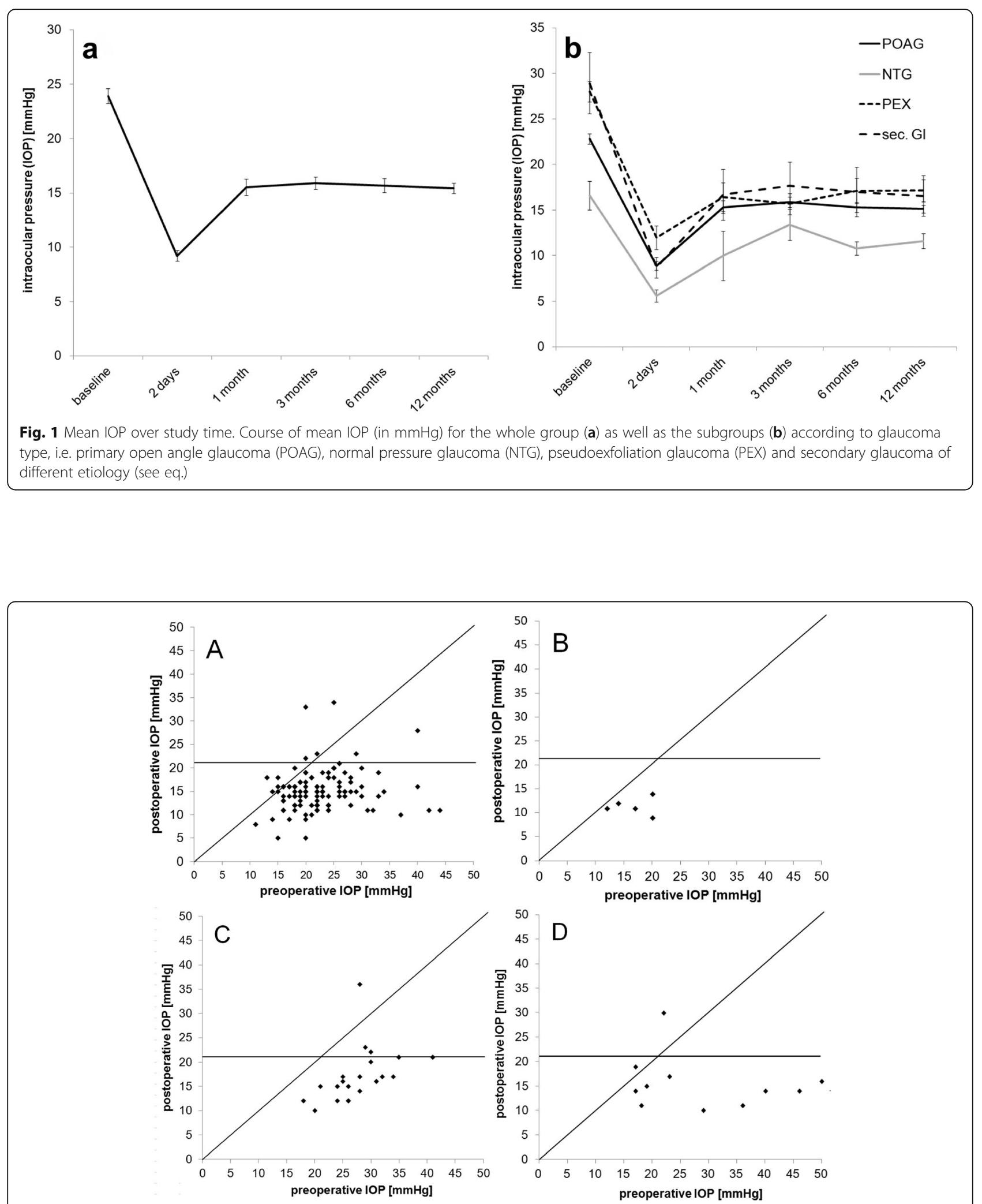

Fig. 2 Pre- and postoperative IOP in different groups of glaucoma. Scattergram of pre- and 12-month postoperative IOP (in $\mathrm{mmHg}$ ) in the four subgroups of glaucoma treated with XEN-microstent-implantation in (a) primary open angle glaucoma, (b) normal pressure glaucoma, (c) pseudoexfoliation glaucoma, (d) secondary glaucoma. The $21 \mathrm{mmHg}$-value is highlighted with a horizontal line to recognize the proportion of cases with an IOP below or above $21 \mathrm{mmHg} 12$ months after surgery 
Table 2 Mean IOP in different groups of glaucoma at all examination time points

\begin{tabular}{lllllllll}
\hline & baseline & 2 days & 1 month & 3 months & 6 months & 12 months & $\boldsymbol{p =}$ & mean reduction [\%] \\
\hline complete group & $23.9 \pm 7.4$ & $9.2 \pm 5.2$ & $15.5 \pm 7.9$ & $15.9 \pm 6.1$ & $15.7 \pm 6.7$ & $15.4 \pm 5.1$ & $<\mathbf{0 . 0 1}$ & $-31.3 \pm 23.9$ \\
POAG & $22.8 \pm 6.5$ & $8.9 \pm 5.1$ & $15.3 \pm 7.6$ & $15.9 \pm 6.0$ & $15.3 \pm 6.2$ & $15.1 \pm 4.6$ & $<\mathbf{0 . 0 1}$ & $-29.8 \pm 23.8$ \\
NTG & $16.6 \pm 3.4$ & $5.6 \pm 2.7$ & $10.0 \pm 3.0$ & $13.4 \pm 2.9$ & $10.8 \pm 4.6$ & $11.6 \pm 2.2$ & $<\mathbf{0 . 0 5}$ & $-28.6 \pm 18.4$ \\
PEX & $28.0 \pm 7.9$ & $11.9 \pm 6.4$ & $16.5 \pm 8.0$ & $15.7 \pm 6.2$ & $17.1 \pm 7.2$ & $17.1 \pm 6.6$ & $<\mathbf{0 . 0 1}$ & $-37.8 \pm 17.9$ \\
secondary glaucoma & $28.9 \pm 13.9$ & $8.7 \pm 4.6$ & $16.7 \pm 10.6$ & $17.7 \pm 10.3$ & $17.0 \pm 10.4$ & $15.5 \pm 6.9$ & $<\mathbf{0 . 0 5}$ & $-35.7 \pm 36.3$
\end{tabular}

Mean IOP $(\mathrm{mmHg})$ measured at baseline and all follow-ups after XEN microstent-implantation. Results are reported for the whole group of eyes treated and for the four subgroups of eyes with different glaucoma types. The indicated $P$-values and mean reduction represent the comparison between the results of the baseline examination and the 12-month results of the respective group

IOP dropped to $8.7 \pm 4.6 \mathrm{mmHg} 2$ days after surgery. Thereafter, as in the other treatment groups, mean IOP increased to $16.7 \pm 10.6 \mathrm{mmHg} 1$ month after XEN implantation and remained stable with a mean of $15.5 \pm$ $6.9 \mathrm{mmHg}$ at the follow-up examination 1 year after surgery, which corresponds to a mean IOP decrease of $36 \%$ compared to baseline. Comparison of follow-up to baseline results again showed a difference of statistical significance at all-time points $(p<0.05)$.

Further comparison of IOP results between the four treatment groups 12 months after XEN microstent implantation also revealed differences of statistical significance (Kruskal-Wallis: $p<0.01$ ). Here further testing revealed differences of statistical significance between POAG and NTG groups $(p=0.029)$ as well as between NTG and PEX groups $(p=0.006)$. However, when comparing results for mean IOP reduction from baseline to 12 months after surgery between the four groups, no difference of statistical significance was found (KruskalWallis: $p=0.2$ ).

\section{Complete and qualified success}

Taking into account the WGA guidelines and the criteria summarized above defining complete and qualified success levels $A$ to $C$, in the complete group of 153 eyes $60 \%$ achieved a complete success (A) and $74 \%$ a qualified success (A). Complete success levels $B$ and $C$ were achieved by 51 and $45 \%$ respectively. Qualified success B and $C$ was achieved by 61 and $56 \%$ in the complete group of 153 eyes operated. The exact results for complete and qualified success levels $\mathrm{A}$ to $\mathrm{C}$ achieved in the four subgroups of different glaucoma entities is summarized in Table 3. Further analysis revealed differences of statistical significance between the four groups treated only concerning complete success (A) (Kruskal-Wallis: $p=0.03$ ) and qualified success (C) (Kruskal-Wallis: $p=$ 0.01 ). Further testing revealed that the aforementioned differences of statistical significance were due to higher success rates in the PEX group than in the POAG group (Mann-Whitney test: POAG PEX for complete success (A) $p=0.004$ and POAG PEX for qualified success (C) $p=0.001)$.

\section{IOP-lowering medication}

The exact course of the number of IOP-lowering eye drops applied is summarized in Fig. 3 and Table 4. The average number of active substances applied in the entire group of 153 eyes fell from $2.6 \pm 1.2$ before to $0.8 \pm 1.312$ months after XEN implantation $(p<$ 0.01 ). In the four subgroups treated the number of applied drugs decreased from $2.5 \pm 1.2$ to $0.8 \pm 1.3$ in the POAG group $(p<0.01)$, from $2.6 \pm 0.9$ to $0.6 \pm 0.9$ in the NTG group $(p<0.05)$, from $2.9 \pm 1.2$ to $1.0 \pm$ 1.3 in the PEX group $(p<0.01)$ and from $2.7 \pm 0.9$ to $0.7 \pm 1.4$ in the group of eyes suffering from secondary glaucoma $(p<0.01)$ during the first 12 months after surgery.

Table 3 Clinical success rates for different groups of glaucoma

\begin{tabular}{|c|c|c|c|c|c|c|}
\hline & $\begin{array}{l}\text { Complete } \\
\text { success (A) }\end{array}$ & $\begin{array}{l}\text { Qualified } \\
\text { success (A) }\end{array}$ & $\begin{array}{l}\text { Complete } \\
\text { success (B) }\end{array}$ & $\begin{array}{l}\text { Qualified } \\
\text { success (B) }\end{array}$ & $\begin{array}{l}\text { Complete } \\
\text { success (C) }\end{array}$ & $\begin{array}{l}\text { Qualified } \\
\text { success (C) }\end{array}$ \\
\hline complete group & $60 \%$ & $74 \%$ & $51 \%$ & $61 \%$ & $45 \%$ & $56 \%$ \\
\hline POAG & $54 \%$ & $71 \%$ & $45 \%$ & $57 \%$ & $29 \%$ & $38 \%$ \\
\hline NTG & $60 \%$ & $60 \%$ & $60 \%$ & $60 \%$ & $20 \%$ & $20 \%$ \\
\hline PEX & $86 \%$ & $91 \%$ & $77 \%$ & $82 \%$ & $50 \%$ & $59 \%$ \\
\hline secondary glaucoma & $75 \%$ & $75 \%$ & $58 \%$ & $58 \%$ & $50 \%$ & $50 \%$ \\
\hline Kruskal-Wallis test & $p=0.03$ & $p=0.22$ & $p=0.07$ & $p=0.17$ & $p=0.17$ & $p=0.01$ \\
\hline
\end{tabular}

Clinical Success defined as complete (without the use of IOP-lowering drugs) and qualified (with the use of additional IOP-lowering drugs) success for the complete treated group of eyes and the four subgroups of the different glaucoma types (A) IOP reduction of $\geq 20 \%$ from baseline and resulting IOP of $<21$ $\mathrm{mmHg}$; complete success (B) IOP reduction of $\geq 30 \%$ from baseline and resulting IOP of $<18 \mathrm{mmHg}$; complete success (C) IOP reduction of $\geq 40 \%$ from baseline and resulting IOP of $<15 \mathrm{mmHg}$ ). Additionally, distribution of achieved success levels was compared between treatment groups using Kruskal-Wallis test 


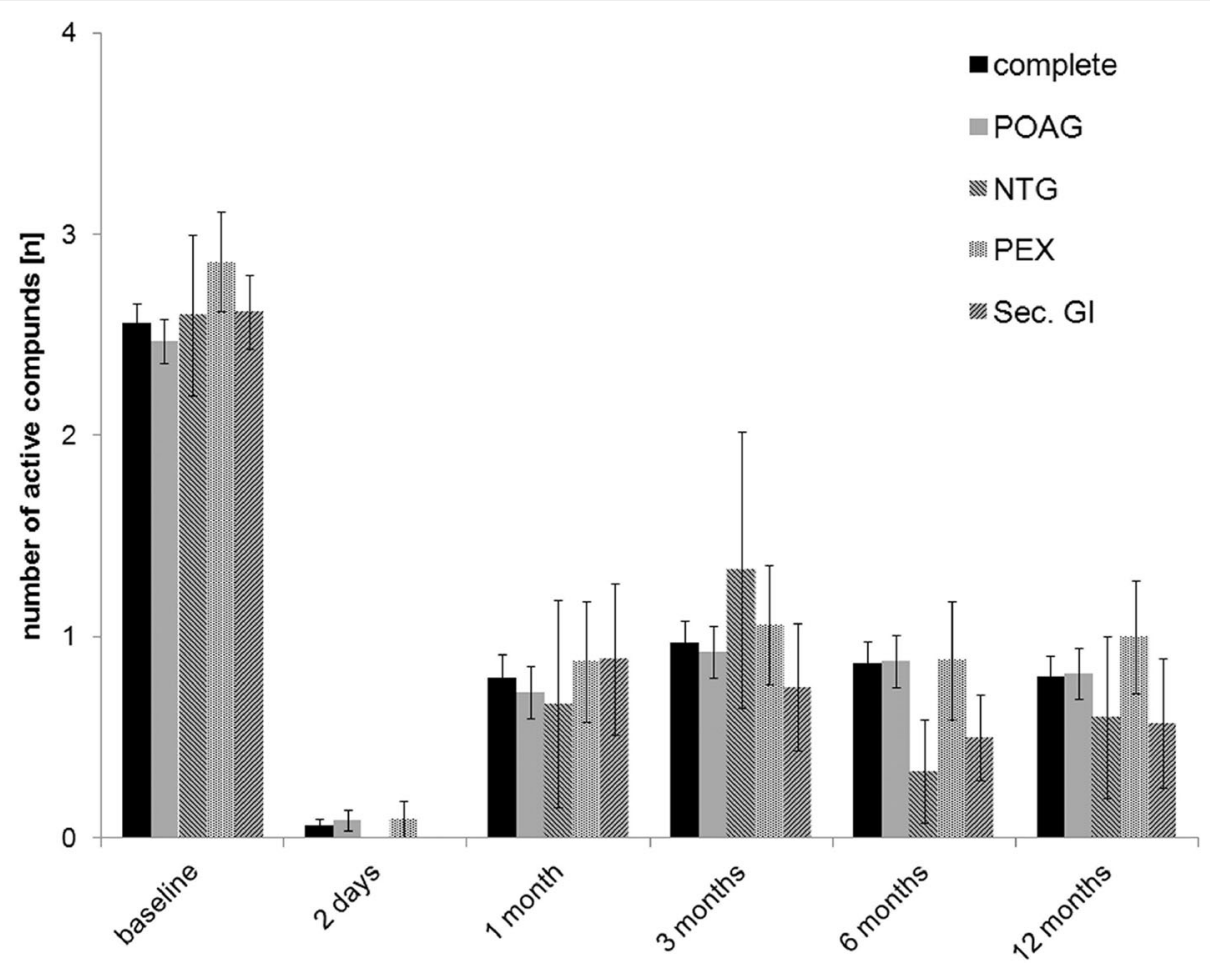

Fig. 3 IOP-lowering agents over study time. Progression of the number of mean IOP-lowering agents (number) used in the entire XEN Microstent patient group and in the subgroups depending on glaucoma type during the first 12 months of postoperative follow-up. (primary open angle glaucoma (POAG); normal pressure glaucoma (NTG); pseudoexfoliation glaucoma (PEX); secondary glaucoma (sec. Gl))

\section{Visual acuity}

In the entire group of 153 eyes treated with the XEN microstent, mean BCVA remained stable and showed no difference of statistical significance during postoperative follow-up. Mean BCVA was $0.38 \pm 0.52 \operatorname{logMAR}$ before surgery and $0.36 \pm 0.49 \log$ MAR 6 months and $0.37 \pm$ $0.49 \log$ MAR 12 months after surgery $(p=0.97$ after 6 months and $p=0.88$ after 12 months compared to baseline) (See also Fig. 4 for the exact course).

In the 113 POAG eyes mean BCVA was $0.33 \pm 0.47$ $\log$ MAR before and $0.34 \pm 0.47 \log M A R 12$ months after surgery $(p=0.31)$. This included 7 eyes with a BCVA loss of $\geq 0.2$ lines during the 12 months of follow-up. Reasons for this loss of function was glaucoma disease progression in 2 cases, AMD progression in 3 cases,
CSME development in 1 case and further progression of senile cataract in 1 case. In the $5 \mathrm{NTG}$ eyes mean BCVA was $0.42 \pm 0.46 \log$ MAR before and $0.36 \pm 0.37 \log$ MAR 12 months after surgery $(p=0.22)$. In the NTG group no case of BCVA loss of $\geq 0.2$ lines was registered during follow-up. In the 13 PEX eyes mean BCVA was $0.36 \pm$ $0.46 \log$ MAR before and $0.22 \pm 0.23 \log$ MAR 12 months after surgery $(p=0.92)$. In the PEX group one case of vision loss of $\geq 0.2$ lines was registered, which was due to progression of glaucoma. In the group of eyes treated for secondary glaucoma mean BCVA was $0.74 \pm 0.63 \log$ MAR before and $0.66 \pm 0.53 \operatorname{logMAR} 12$ months after surgery $(p=0.91)$. This included one case experiencing vision loss of $\geq 0.2$ lines, which was due to progression of glaucoma.

Table 4 Mean IOP lowering agents in different groups of glaucoma at all examination time points

\begin{tabular}{|c|c|c|c|c|c|c|c|}
\hline & baseline & 2 days & 1 month & 3 months & 6 months & 12 months & $p=$ \\
\hline complete group & $2.6 \pm 1.2$ & $0.1 \pm 0.5$ & $0.8 \pm 1.4$ & $1.0 \pm 1.4$ & $0.9 \pm 1.4$ & $0.8 \pm 1.3$ & $<0.01$ \\
\hline POAG & $2.5 \pm 1.2$ & $0.1 \pm 0.5$ & $0.7 \pm 1.4$ & $0.9 \pm 1.4$ & $0.9 \pm 1.4$ & $0.8 \pm 1.3$ & $<0.01$ \\
\hline NTG & $2.6 \pm 0.9$ & $0 \pm 0$ & $0.7 \pm 1.2$ & $0.7 \pm 1.0$ & $0.3 \pm 0.6$ & $0.6 \pm 0.9$ & $<0.05$ \\
\hline PEX & $2.9 \pm 1.2$ & $0.1 \pm 0.4$ & $0.9 \pm 1.4$ & $1.1 \pm 1.4$ & $0.9 \pm 1.4$ & $1.0 \pm 1.3$ & $<0.01$ \\
\hline secondary glaucoma & $2.6 \pm 0.9$ & $0 \pm 0$ & $0.9 \pm 1.8$ & $0.8 \pm 1.5$ & $0.5 \pm 1.0$ & $0.6 \pm 1.5$ & $<0.01$ \\
\hline
\end{tabular}

Examination values for the number of applied IOP-lowering agents at the beginning of the treatment as well as for all follow-ups after XEN implantation for the whole treated group of eyes and the four subgroups of the different glaucoma types. The $P$-values given represent the comparison between the baseline and $12-$ month results for each group 


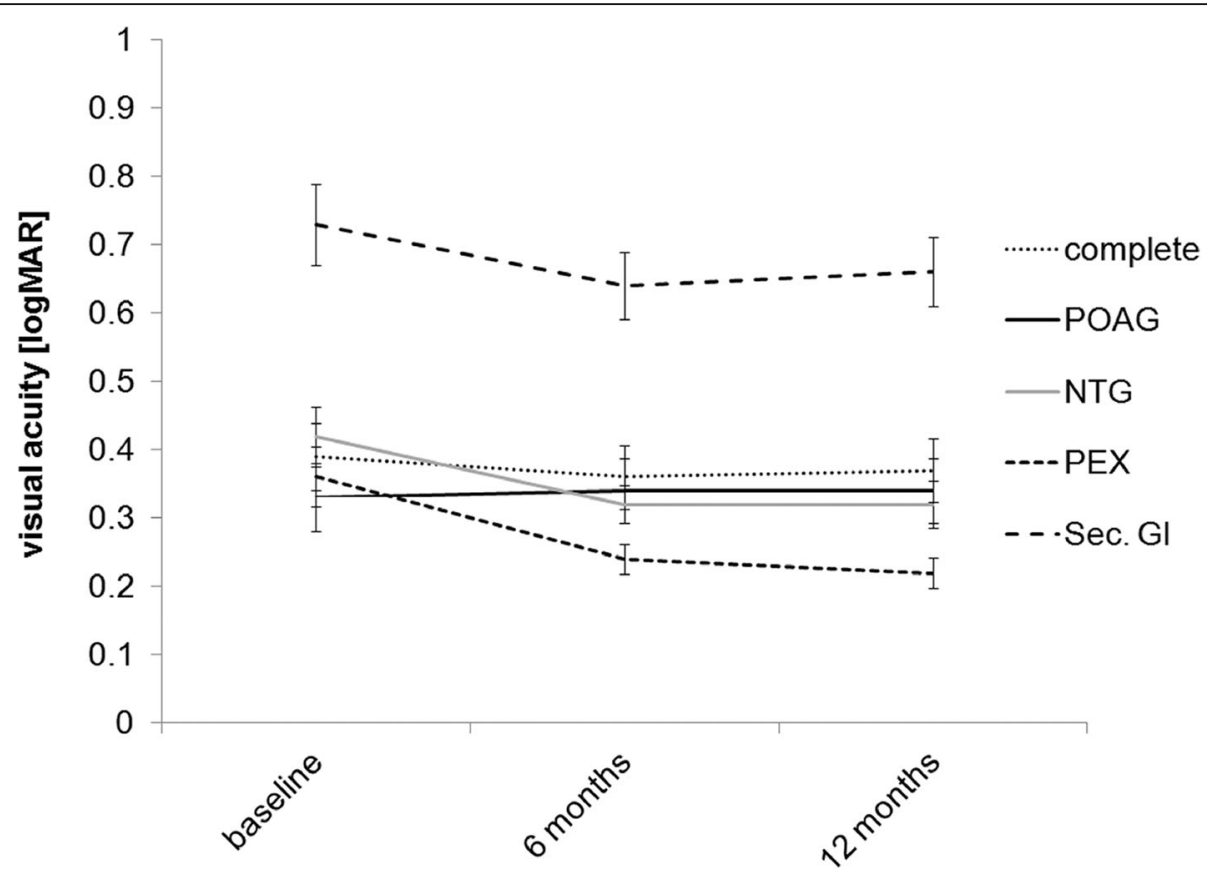

Fig. 4 Visual acuity over study time in different groups of glaucoma. Course of BCVA in the entire group of eyes treated with XEN microstent implantation and in subgroups depending on the type of glaucoma in the first 12 months of postoperative follow-up.(primary open angle glaucoma (POAG); normal pressure glaucoma (NTG); pseudoexfoliation glaucoma (PEX); secondary glaucoma (sec. Gl))

Comparison of BCVA between groups showed that there were differences of statistical significance concerning BCVA (Kruskal-Wallis: $p<0.01$ ). Mean BCVA was worse in the secondary glaucoma group than in the three other groups treated. Further performed MannWhitney tests confirmed a difference of statistical significance when comparing BCVA between the secondary glaucoma and the POAG groups $(p<0.01)$ and between the secondary glaucoma and the PEX groups $(p<0.01)$. Further testing did not reveal differences of statistical significance between any of the other groups. Finally, when comparing the BCVA change occurring over the 12 month follow-up period in between the four groups treated no difference of statistical significance could be revealed (Kruskall-Wallis; $p=0.35$ ).

Table 5 Visual field defects at different time points in different groups of glaucoma

\begin{tabular}{lllll}
\hline & baseline & $\mathbf{6}$ months & 12 months & $\boldsymbol{p =}$ \\
\hline complete group & $10.1 \pm 4.3$ & $10.8 \pm 4.3$ & $10.8 \pm 4.2$ & 0.11 \\
POAG & $10.2 \pm 3.9$ & $10.6 \pm 3.9$ & $10.5 \pm 3.9$ & 0.08 \\
NTG & $10.9 \pm 2.9$ & $10.8 \pm 2.3$ & $10.8 \pm 2.5$ & 0.23 \\
PEX & $9.7 \pm 4.7$ & $9.8 \pm 4.5$ & $9.6 \pm 4.7$ & 0.17 \\
secondary glaucoma & $10.0 \pm 4.9$ & $10.7 \pm 5.0$ & $10.7 \pm 5.0$ & 0.37 \\
\hline
\end{tabular}

Examination values for mean deviation (in decibels) of automated standard perimetry at baseline and follow-up 6 and 12 months after XEN implantation for the entire patient population and the four subgroups of different glaucoma types. The $P$-values given represent the comparison between the results at baseline and at 12 months for each group and do not show any statistically significant difference for any of the groups analyzed

\section{Visual field}

Simultaneously with the drop of mean IOP, mean visual field defect remained stable. In the complete group of 153 eyes treated mean defect was $10.1 \pm 4.3 \mathrm{~dB}$ at baseline, increased slightly to $10.8 \pm 4.3 \mathrm{~dB}$ at 6 months ( $p=$ 0.11 ) and was $10.8 \pm 4.2 \mathrm{~dB}$ at 12 months after surgery, which was not statistically significant $(p=0.15)$. Also, the comparison of mean BCVA between the four treatment groups before and 12 months after surgery did not

Table 6 Needling rates and complications

\begin{tabular}{ll}
\hline needling procedures & 64 in 54 eyes \\
& POAG: 38 of $113(34 \%)$ \\
& NTG: 1 of $5(20 \%)$ \\
& PEX: 12 of $22(55 \%)$ \\
& Sec. Gl.: 3 of $13(23 \%)$ \\
& 20 of $153(13 \%)$ \\
choroidal detachment & 12 of $153(8 \%)$ \\
prominent or large subconjunctival hemorrhage & 10 of $153(7 \%)$ \\
hyphaema & 3 of $153(2 \%)$ \\
shallow anterior chamber & 3 of $153 \%(2 \%)$ \\
macular edema & 2 of $153(1 \%)$ \\
conjunctival erosion & 1 of $153(<1 \%)$ \\
uveitis & 1 of $153(<1 \%)$ \\
keratitis & 1 of $153(<1 \%)$ \\
corneal erosion & 1 as for $1 \%$ four
\end{tabular}

Needling rate for the whole group of patients (as well as for the four subgroups of the different types of glaucoma treated) and the rate of other complications observed during the first 12 months of follow-up after XEN microstent implantation in uncontrolled glaucoma in all groups together 
reveal differences of statistical significance (KruskalWallis; before surgery: $p=0.08 ; 12$ months: $p=0.3$ ) (see Table 5 for details).

\section{Complications and needling rate}

To achieve the results described, 64 needling procedures had to be performed in 54 of the 153 eyes (35.3\%). 44 eyes needed 1 needling and another 10 eyes needed 2 needlings during the first year after XEN implantation. None of the eyes needed $>2$ needlings. The needling rate was different between the four subgroups analyzed (see Table 6). The lowest needling rate was found in NTG eyes $(20 \%)$ and the highest rate in PEX glaucoma eyes (55\%). In the total group of 153 eyes treated 20 eyes showed benign choroidal detachment during the first postoperative month. All cases responded well to conservative treatment (contact lens and cycloplegia). A large and prominent subconjunctival hemorrhage (to the extent that it was classified as clinically significant) was seen in 12 cases immediately after surgery, but was resorbed after 1 to 4 weeks. Blood in the anterior chamber immediately after surgery was seen in 10 eyes and dissolved in all cases without further consequences after 1 to 4 weeks. Postoperative macular edema was seen in 3 eyes, which all responded well to treatment with carbonic anhydrase inhibitors. Apart from the above described incidents, no serious complications were observed in the treated eyes.

\section{Discussion}

Classic TE with or without the use of antimetabolites is the "gold standard" for surgical treatment of a wide range of glaucoma entities. The XEN microstent is designed to bypass the transtrabecular outflow pathway whereby reducing IOP. Since its initial description, a number of reports have been published on the efficacy and safety profile of the XEN microstent for the treatment of POAG [18, 23, 24]. In summary, implantation of the XEN microstent can significantly reduce IOP and the amount of IOP-lowering eye drops applied, resulting in acceptable success rates in POAG after 12 and 24 months. Reitsamer and colleagues already showed in their one and two-year multicenter study (APEX study) that IOP and required medication can be reduced effectively by implantation of the XEN microstent either alone or in combination with phacoemulsification and posterior chamber lens implantation [23] Some groups even reported on achieving lower mean postoperative IOP values of around $12 \mathrm{mmHg}$ when starting from a lower initial mean level at baseline before surgery [25].

Grover et al. reported on their 12-month single XEN procedure results in a group of 65 eyes with uncontrolled and already pre-operated glaucoma cases $(85 \%$ of eyes had already been unsuccessfully treated with filtering surgery and/or cilioablative techniques) [26] The results were similar to the data hereby reported with a qualified success rate of $75 \%$, a reduction of mean IOP from $25.1 \pm 3.7 \mathrm{mmHg}$ at baseline to $15.9 \pm 5.2$ mmHg 12 months after surgery and a needling rate of about $32 \%$. The patient cohort reported on also included a large percentage of POAG cases and some PEX cases, but no case of secondary glaucoma. Grover et al. also did not report on the efficacy of the XEN microstent in subgroups of different glaucoma entities. Lenzhofer et al. already demonstrated the long-term effectiveness of the XEN microstent in a group of 64 eyes of 64 POAG patients. It was shown that low mean IOP values and reduced necessity for application of IOP-lowering medication can even be found as long as 4 years after implantation of the XEN microstent [27]. However, these data were collected in eyes in which the XEN 63 was implanted (with a larger internal lumen of $63 \mu \mathrm{m}$ ). Recently Fea et al. reported a prospective multicenter study with different types of glaucoma in 115 patients, 56 patients got combined surgery with phacoemulsification and the follow up was 12 months [28]. Mean IOP and medications decreased significantly until study end point. An IOP reduction of $20 \%$ was achieved in 72,3 and $30 \%$ reduction in $52.6 \%$.

Given the mode of action utilized, the XEN microstent should in theory also be applicable in glaucoma entities other than POAG. Although some groups reported on the treatment of different types of glaucoma with the XEN microstent, they so far failed analyzing the efficacy in the treated subgroups $[24,29]$. We analyzed the 12 month postoperative follow-up results in NTG, PEX and secondary glaucoma cases and compared the results with those obtained in a medium-sized comparison group of POAG eyes. With the reported postoperative results we could clearly and for the first time demonstrate that IOP and necessary IOP-lowering medication can be lowered with a comparable efficacy in NTG, PEX and secondary glaucoma cases as in POAG. Apart from this visual acuity and visual field indices remained stable without evidence for further postoperative deterioration, although only the first 12 months after surgery were monitored. There was a notable difference in mean BCVA, which was worse but also stable during the postoperative course in the subgroup of treated secondary glaucoma cases. This must be interpreted within the context of the underlying disease process leading to secondary glaucoma, which in most cases has devastating consequences on visual acuity.

Postoperative results after implantation of the XEN microstent other types of glaucoma than POAG have also been described by other authors. Ibanez-Munoz and colleagues implanted the XEN microstent in 36 eyes with POAG and 37 eyes with secondary glaucoma, 34 of 
which had PEX glaucoma [30]. The difference in the postoperative results regarding the reduction of IOP and the required medication was not statistically significant in both groups. Apart from that, no difference was found when the XEN microstent was implanted as a solo procedure or in combination with cataract extraction and IOL implantation.

Publications on study data for 1 year and longer from single XEN microstent implantations without phacoemulsification are rare. Most studies combine groups with and without phacoemulsification. The work from Grover et al. was already shown [26]. Tan et al. presented a retrospective interventinal case series of 39 eyes, some with previous glaucoma surgery [31]. With smaller different glaucoma groups than this study eyes showed in mean an IOP reduction from 24.9 to $14.5 \mathrm{mmHg}$ with a decrease from 3 medications pre-surgery to 0,7 at 12 months. Complete Success was achieved in 87\% (definition A) and 62\% (definition B) while qualified success was 92 and $64 \%$ respectively.

Karimi et al. (like our group) have implanted the XEN microstent in different glaucoma types, including traumatic and neovascular glaucoma, but have not reported on postoperative results in these treated subgroups [32]. They also found no statistically significant differences in their results regarding IOP reduction and medication in cases where the XEN microstent was implanted alone or in combination with cataract extraction. In the 259 eyes treated, $41 \%$ required needling during the first 18 months after implantation. However, it should be noted that results originating from only 34 and $12 \%$ of eyes were included for analysis of the 12- and 18-month postoperative follow-up.

The higher rate of needling after XEN microstent implantation compared to TE, the "gold standard" in glaucoma surgery, has been the subject of criticism in the past. Schlenker et al. and others reported on further necessary secondary interventions and safety problems when comparing the results following both XEN microstent implantation and $\mathrm{TE} \pm \mathrm{MMC}$, which showed a slightly higher needling rate after XEN, but which did not reach statistical significance during further analysis [33]. However, the different rates of necessary postoperative interventions and the rates of postoperative complications were not statistically significant between the groups treated with XEN microstent or TE. In summary, XEN microstent and TE are comparable in terms of postoperative safety. However TE led to more visits, especially in the first 3 months after surgery. As shown in our study PEX eyes had the highest needling rates but also the highest success rates. This was also confirmed in several studies so far as shown in the comprehensive recent review from Fea et al. [20] Success rates for XEN microstent as single procedure in uveitic glaucoma showed good results in a small number of patients in several studies from up to 60 to $65 \%$ reduction of IOP and significant medication lowering. Comparison for NTG are missing [20].

The main limitation of this study is that although the number of patients with other forms of glaucoma is comparatively small. This is usually the case in most studies because of the lower incidence of these glaucoma entities. Only eyes suffering from a medium to high glaucoma damage were included in this study. Further studies should focus on early stages of glaucoma, since the minimally invasive approach of MIGS would be interesting for early surgical intervention. Lowering IOP to mean values around $15 \mathrm{mmHg}$ on average certainly represents significant reduction, but according to the advanced glaucoma intervention study (AGIS) is certainly no guarantee to prevent progression [34]. Lowering intraocular pressure to less than $12 \mathrm{mmHg}$ would be necessary here and was not achieved in all eyes. An additional reduction of intraocular pressure by medication or surgery has to be discussed in case of further disease progression.

Contrary to many other studies, this analysis shows a high number of patients outside the classic POAG spectrum. In addition, all patients in this study were followed up exclusively with XEN Micro Stent Implantation as a single surgical procedure with detailed followup of various glaucoma parameters over 12 months. In this analysis only one eye per patient was included (even if both eyes were treated). And for all included eyes a full 12-month postoperative follow-up was available. One limiting factor, concerning the hereby presented data, is that the sizes of the four treatment groups were different. However, the largest group, which comprises POAG patients, should only be regarded as a comparison group for the other, less frequent glaucoma entities, treated under the same conditions. In the entire study group, XEN microstent implantation not only resulted in a reduction of IOP and necessary IOP-lowering drugs, but also had no significant effect on BCVA, neither in the total group of 153 eyes nor in the four subgroups analyzed. Lenzhofer et al. showed similar results in their treated POAG cases [35]. It has also been shown earlier that BCVA in eyes after TE decreases, even when taking into account (and ruling out) the known side effect of cataract formation [36].. Therefore, our hereby presented data also complement the growing evidence in favor for the XEN microstent in POAG and other types of glaucoma with an unblocked anterior chamber angle.

\section{Conclusion}

Implantation of the XEN microstent was effective in reducing intraocular pressure and the number of drugs applied to reduce intraocular pressure, while BCVA and 
perimetry results remained unchanged. In conclusion, the data presented here on the efficacy and safety of the XEN microstent in glaucoma types other than POAG are encouraging for the use of this (comparatively new) technique also in the treatment of PEX and other secondary glaucoma cases. Longer follow-up time, a larger number of eyes treated and a comparison group comparing the efficacy of the XEN microstent to the "gold standard" of glaucoma surgery, which remains the TE, are necessary to further demonstrate the usefulness of this technique.

\section{Abbreviations \\ 5-FU: 5-Fluoruracil; BCVA: Best corrected visual acuity; IOP: Intraocular pressure; MD: Mean deviation; MIGS: Minimally invasive glaucoma surgery; MMC: Mitomycin C; NTG: Normal-tension glaucoma; PEX: Pseudoexfoliation glaucoma; POAG: Primary open angle glaucoma; RNFL: Retinal nerve fiber layer; RNFL-OCT: Retinal nerve fiber layer thickness - optical coherence tomography; Sec.GI: Secondary glaucoma; TE: Trabeculectomy}

\section{Acknowledgements}

None.

\section{Authors` contributions}

Involved in the design of the study (JDU,MS,MR,TT); conduct of the study (JDU,TT,CBU,CBO); collection, management, analysis of the data (JDU,TT,CBU,CBO); preparation of the manuscript (JDU,TT,MS,CBU,CBO,MR); and critical revision of the manuscript (JDU,TT,MS,CBU,CBO,MR). All authors read and approved the final manuscript.

\section{Funding}

The publication fees were paid by the Open Access Publication Fund of the University of Leipzig. The funding bodies played no role in the design of the study and collection, analysis, and interpretation of data and in writing the manuscript. Open Access funding enabled and organized by Projekt DEAL.

\section{Availability of data and materials}

The datasets obtained during and/or analyzed during the current study available from the corresponding author on reasonable request.

\section{Ethics approval and consent to participate}

All procedures performed in studies involving human participants were in accordance with the ethical standards of the institutional and/or national research committee and with the 1964 Helsinki declaration and its later amendments or comparable ethical standards. (Ethics approval: No: 209-18ek (Ethics committee of medical faculty of University of Leipzig, Leipzig, Germany)) Written informed consent was obtained from all individual participants included in the study.

\section{Consent for publication}

Not Applicable.

\section{Competing interests}

The authors declare that they have no conflict of interest.

\section{Author details}

'Department of Ophthalmology, Heinrich-Heine-University, Duesseldorf, Germany. ${ }^{2}$ Department of Ophthalmology, University Eye Hospital, University of Leipzig, Liebigstrasse 10-14, 04103 Leipzig, Germany.

Received: 24 June 2020 Accepted: 14 December 2020

Published online: 17 December 2020

\section{References}

1. Quigley HA. Number of people with glaucoma worldwide. Br J Ophthalmol. 1996;80(5):389-93. https://doi.org/10.1136/bjo.80.5.389.

2. Tham YC, Li X, Wong TY, Quigley HA, Aung T, Cheng CY. Global prevalence of glaucoma and projections of glaucoma burden through 2040: a systematic review and meta-analysis. Ophthalmology. 2014;121(11):2081-90. https://doi.org/10.1016/j.ophtha.2014.05.013.

3. Quigley HA. Ganglion cell death in glaucoma: pathology recapitulates ontogeny. Aust N Z J Ophthalmol. 1995;23(2):85-91.

4. Levkovitch-Verbin H. Retinal ganglion cell apoptotic pathway in glaucoma: initiating and downstream mechanisms. Prog Brain Res. 2015;220:37-57. https://doi.org/10.1016/bs.pbr.2015.05.005.

5. Mitchell P, Hourihan F, Sandbach J, Wang JJ. The relationship between glaucoma and myopia: the Blue Mountains eye study. Ophthalmology. 1999;106(10):2010-5. https://doi.org/10.1016/s0161-6420(99)90416-5.

6. Weinreb RN, Khaw PT. Primary open-angle glaucoma. Lancet. 2004; 363(9422):1711-20. https://doi.org/10.1016/S0140-6736(04)16257-0S01406736(04)16257-0.

7. Leske MC, Wu SY, Hennis A, Honkanen R, Nemesure B. Risk factors for incident open-angle glaucoma: the Barbados eye studies. Ophthalmology. 2008;115(1):85-93. https://doi.org/10.1016/j.ophtha.2007.03.017.

8. Heijl A, Leske MC, Bengtsson B, Hyman L, Hussein M. Reduction of intraocular pressure and glaucoma progression: results from the early manifest Glaucoma trial. Arch Ophthalmol. 2002;120(10):1268-79.

9. Leske MC, Heijl A, Hussein M, Bengtsson B, Hyman L, Komaroff E. Factors for glaucoma progression and the effect of treatment: the early manifest glaucoma trial. Arch Ophthalmol. 2003;121(1):48-56.

10. Schmidl D, Schmetterer L, Garhofer G, Popa-Cherecheanu A. Pharmacotherapy of glaucoma. J Ocul Pharmacol Ther. 2015;31(2):63-77. https://doi.org/10.1089/jop.2014.0067.

11. Landers J, Martin K, Sarkies N, Bourne R, Watson P. A twenty-year follow-up study of trabeculectomy: risk factors and outcomes. Ophthalmology. 2012; 119(4):694-702. https://doi.org/10.1016/j.ophtha.2011.09.043.

12. Razeghinejad MR, Spaeth GL. A history of the surgical management of glaucoma. Optom Vis Sci. 2011;88(1):E39-47. https://doi.org/10.1097/OPX. Ob013e3181fe2226.

13. Zahid S, Musch DC, Niziol LM, Lichter PR. Risk of endophthalmitis and other long-term complications of trabeculectomy in the collaborative initial Glaucoma treatment study (CIGTS). Am J Ophthalmol. 2013;155(4):674-680, 680 e671. https://doi.org/10.1016/j.ajo.2012.10.017.

14. Kim EA, Law SK, Coleman AL, Nouri-Mahdavi K, Giaconi JA, Yu F, Lee JW, Caprioli J. Long-term bleb-related infections after trabeculectomy: incidence, risk factors, and influence of bleb revision. Am J Ophthalmol. 2015;159(6): 1082-91. https://doi.org/10.1016/j.ajo.2015.03.001.

15. Picht G, Mutsch Y, Grehn F. Follow-up of trabeculectomy. Complications and therapeutic consequences. Ophthalmologe. 2001;98(7):629-34. https:// doi.org/10.1007/s003470170098.

16. Picht G, Grehn F. Development of the filtering bleb after trabeculectomy. Classification, histopathology, wound healing process. Ophthalmologe. 1998;95(5):W380-7.

17. Lavia C, Dallorto L, Maule M, Ceccarelli M, Fea AM. Minimally-invasive glaucoma surgeries (MIGS) for open angle glaucoma: a systematic review and meta-analysis. PLoS One. 2017;12(8):e0183142. https://doi.org/10.1371/ journal.pone.0183142 PONE-D-16-37826.

18. Sheybani A, Lenzhofer M, Hohensinn M, Reitsamer H, Ahmed II. Phacoemulsification combined with a new ab interno gel stent to treat open-angle glaucoma: pilot study. J Cataract Refract Surg. 2015;41(9):19059. https://doi.org/10.1016/j.jcrs.2015.01.019.

19. Sheybani A, Reitsamer H, Ahmed II. Fluid dynamics of a novel micro-fistula implant for the surgical treatment of Glaucoma. Invest Ophthalmol Vis Sci. 2015;56(8):4789-95. https://doi.org/10.1167/iovs.15-16625.

20. Fea AM, Durr GM, Marolo P, Malinverni L, Economou MA, Ahmed I. XEN • Gel Stent: A Comprehensive Review on Its Use as a Treatment Option for Refractory Glaucoma. 2020;14:1805-32.

21. Theilig T, Rehak M, Busch C, Bormann C, Schargus M. Unterlauft JD (2020) comparing the efficacy of trabeculectomy and XEN gel microstent implantation for the treatment of primary open-angle glaucoma: a retrospective monocentric comparative cohort study. Sci Rep. 2020;10(1): 19337. https://doi.org/10.1038/s41598-020-76551-y.

22. Shaarawy T, Grehn F, Sherwood M. WGA guidelines on design and reporting of Glaucoma surgical trials. Amsterdam: Kugler Publications; 2009.

23. Reitsamer H, Sng C, Vera V, Lenzhofer M, Barton K, Stalmans I. Two-year results of a multicenter study of the ab interno gelatin implant in medically uncontrolled primary open-angle glaucoma. Graefes Arch Clin Exp Ophthalmol. 2019:257(5):983-96. https://doi.org/10.1007/s00417-019-04251z10.1007/s00417-019-04251-z. 
24. Mansouri K, Guidotti J, Rao HL, Ouabas A, D'Alessandro E, Roy S, Mermoud A. Prospective evaluation of standalone XEN gel implant and combined phacoemulsification-XEN gel implant surgery: 1-year results. J Glaucoma. 2018;27(2):140-7. https://doi.org/10.1097/JJG.0000000000000858.

25. Galal A, Bilgic A, Eltanamly R, Osman A. XEN Glaucoma implant with Mitomycin C 1-year follow-up: result and complications. J Ophthalmol. 2017; 2017:5457246. https://doi.org/10.1155/2017/5457246.

26. Grover DS, Flynn WJ, Bashford KP, Lewis RA, Duh YJ, Nangia RS, Niksch B. Performance and safety of a new Ab Interno gelatin stent in refractory Glaucoma at 12 months. Am J Ophthalmol. 2017;183:25-36. https://doi.org/ 10.1016/j.ajo.2017.07.023.

27. Lenzhofer M, Kersten-Gomez I, Sheybani A, Gulamhusein H, Strohmaier C, Hohensinn M, Burkhard Dick H, Hitzl W, Eisenkopf L, Sedarous F, Ahmed II, Reitsamer HA. Four-year results of a minimally invasive transscleral glaucoma gel stent implantation in a prospective multi-Centre study. Clin Exp Ophthalmol. 2019;47(5):581-7. https://doi.org/10.1111/ceo.13463.

28. Fea AM, Bron AM, Economou MA, et al. European study of the efficacy of a cross-linked gel stent for the treatment of glaucoma. J Cataract Refract Surg. 2020;46:441-50.

29. Widder RA, Dietlein TS, Dinslage S, Kuhnrich P, Rennings C, Rossler G. The XEN45 gel stent as a minimally invasive procedure in glaucoma surgery: success rates, risk profile, and rates of re-surgery after 261 surgeries. Graefes Arch Clin Exp Ophthalmol. 2018;256(4):765-71. https://doi.org/10.1007/ s00417-018-3899-7.

30. Ibanez-Munoz A, Soto-Biforcos VS, Rodriguez-Vicente L, Ortega-Renedo I, Chacon-Gonzalez M, Rua-Galisteo O, Arrieta-Los Santos A, Lizuain-Abadia ME, Del Rio Mayor JL. XEN implant in primary and secondary open-angle glaucoma: a 12-month retrospective study. Eur J Ophthalmol. 2019: 1120672119845226. https://doi.org/10.1177/1120672119845226.

31. Tan SZ, Walkden A, Au L. One-year result of XEN45 implant for glaucoma: efficacy, safety, and postoperative management. Eye. 2018;32:324-32.

32. Karimi A, Lindfield D, Turnbull A, Dimitriou C, Bhatia B, Radwan M, Gouws $P$, Hanifudin A, Amerasinghe N, Jacob A. A multi-Centre interventional case series of 259 ab-interno Xen gel implants for glaucoma, with and without combined cataract surgery. Eye (Lond). 2019;33(3):469-77. https://doi.org/10. 1038/s41433-018-0243-8.

33. Schlenker MB, Gulamhusein H, Conrad-Hengerer I, Somers A, Lenzhofer M, Stalmans I, Reitsamer H, Hengerer FH, Ahmed IIK. Efficacy, safety, and risk factors for failure of standalone Ab Interno gelatin microstent implantation versus standalone trabeculectomy. Ophthalmology. 2017;124(11):1579-88. https://doi.org/10.1016/j.ophtha.2017.05.004.

34. Anonymous. The advanced Glaucoma intervention study (AGIS): 7. The relationship between control of intraocular pressure and visual field deterioration The AGIS Investigators. Am J Ophthalmol. 2000;130:429-40.

35. Lenzhofer M, Strohmaier C, Hohensinn M, Hitzl W, Steiner V, Baca B, Moussa S, Motloch K, Reitsamer HA. Change in visual acuity 12 and 24 months after transscleral ab interno glaucoma gel stent implantation with adjunctive Mitomycin C. Graefes Arch Clin Exp Ophthalmol. 2019;257(12):2707-15. https://doi.org/10.1007/s00417-019-04452-6.

36. Edmunds B, Thompson JR, Salmon JF, Wormald RP. The National Survey of trabeculectomy. III. Early and late complications. Eye (Lond). 2002;16(3):297303. https://doi.org/10.1038/sj.eye.6700148.

\section{Publisher's Note}

Springer Nature remains neutral with regard to jurisdictional claims in published maps and institutional affiliations.

Ready to submit your research? Choose BMC and benefit from:
- fast, convenient online submission
- thorough peer review by experienced researchers in your field
- rapid publication on acceptance
- support for research data, including large and complex data types
- gold Open Access which fosters wider collaboration and increased citations
- maximum visibility for your research: over 100M website views per year
At BMC, research is always in progress.
Learn more biomedcentral.com/submissions

